\title{
Solução Numérica de Equações Diferenciais Parciais Implícitas de Primeira Ordem
}

\author{
Sergio A. Escobedo Antonio Castelo Filho Marcio Gameiro \\ Departamento de Matemática Aplicada e Estatística, ICMC, USP \\ 13566-590, São Carlos, SP \\ E-mail: saquise@icmc.usp.br castelo@icmc.usp.br gameiro@icmc.usp.br
}

\begin{abstract}
Resumo: As equações diferencias parciais têm origem na modelagem de problemas nas ciências e engenharia, tais como: a equação do calor, equação da onda, equação de Poisson, entre outras. Para muitas destas equações não é tão simples obter uma técnica analítica para achar sua solução e nestes casos é necessário o uso de soluções aproximadas obtidas pelo computador. Existem técnicas tradicionais para solução a numérica de uma grande classe de equações diferenciais, mas quando esta equação está na forma implícita, muitas destas técnicas não podem ser aplicadas, principalmente próximas a singularidades. O objetivo deste trabalho é mostrar um método numérico para a solução de equações diferenciais parciais de primeira ordem implícitas.
\end{abstract}

Palavras-chave: Curva Característica, Solução Característica, Métodos de continuação numérica.

\section{Introdução}

Em geral, referenciar uma equação diferencial parcial faz imaginar algum modelo físico que seja descrito por esta equação e ao mesmo tempo pensa-se em procurar algum método que possa determinar sua solução. Ao longo do tempo muitas pessoas resolveram casos particulares com o objetivo de estabelecer soluções analíticas e numéricas sujeitas a diferentes condições. Além disso, diversos estudos na área concluem que pensar numa solução geral para todos os casos ainda não é factível, mas para um caso específico pode-se obter uma "solução geral" .

Habitualmente se prova a existência de uma solução para depois obter métodos de solução analíticos ou numéricos. Existem métodos analíticos que resolvem alguns tipos de EDPs de primeira ordem, podemos mencionar dentro dos métodos analíticos mais conhecidos o método das características de Cauchy (Figura 1), este método tem uma interpretação geométrica que pode ser aproveitada para implementar uma solução numérica.

Com respeito aos Métodos Numéricos que resolvem equações diferenciais, podemos mencionar que as equações diferenciais parciais de segunda ordem, são as mais estudadas com uma maior importância devido a suas diversas aplicações na Física. Desde o século $X I X$ com o uso dos computadores, tem sido feitos trabalhos com bastante rigor no cálculo computacional para determinar soluções numéricas e representações gráficas.

\section{Exposição do problema}

De acordo com os métodos analíticos mencionados, o método das características tem uma boa interpretação geométrica de como resolver equações diferenciais parciais de primeira ordem. Estabeleceremos agora a quem denominaremos curva característica.

Se a equação diferencial parcial está determinada implicitamente pela equação: 


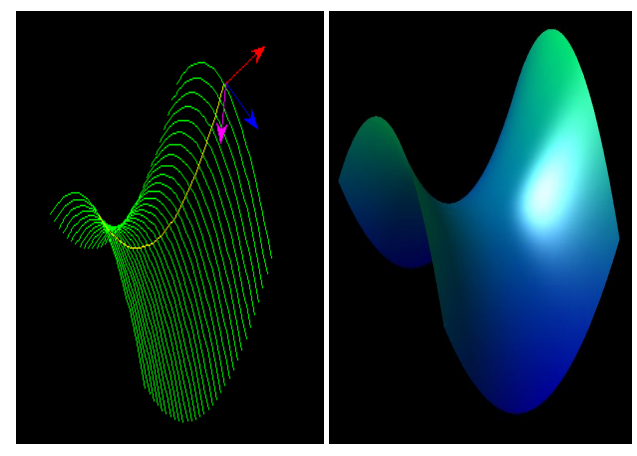

Figura 1: Curvas Características

$$
F\left(x, y, z, z_{x}, z_{y}\right)=0
$$

onde $z=u(x, y)$, e supondo que a curva $\sigma(t)$, dada por $\sigma(t)=(x(t), y(t), z(t), p(t), q(t))$ seja uma curva que verifica $F(\sigma(t))=0$, temos que a projeção dos pontos de $\sigma(t)$ sobre $\mathbb{R}^{3}$ dada por $(x(t), y(t), z(t))$, com vetor normal $N=(p(t), q(t),-1)$, define uma curva, à qual denominaremos, curva solução ou "curva característica" apresentada na Figura 1

Formalizando, consideraremos inicialmente uma equação diferencial parcial de primeira ordem implícita, onde a função solução $u$ esta definida na equação

$$
F\left(x_{1}, x_{2}, u, u_{x_{1}}, u_{x_{2}}\right)=0
$$

tal que $F \in C^{2}(\Omega), \Omega \subset \mathbb{R}^{2} \times \mathbb{R} \times \mathbb{R}^{2}$ é domínio aberto tal que é aplicável o teorema da função implícita com respeito a $u_{x_{1}}$ ou $u_{x_{2}}$.

Denotando $p_{1}=u_{x_{1}}, p_{2}=u_{x_{2}}$ obtemos

$$
F\left(x_{1}, x_{2}, u, p_{1}, p_{2}\right)=0
$$

A função $u$ é definida como $u: V \rightarrow \mathbb{R}, V \subset \mathbb{R}^{2}$ aberto, e o conjunto $\Omega^{*}$ é projeção de $\Omega$ sobre $\mathbb{R}^{3}$ dada por $\left(x_{1}, x_{2}, u\right)$. Assim, pode-se imaginar em $\Omega^{*}$ que o gráfico de $u$ é o conjunto de pontos que tem em cada ponto o vetor normal $\left(p_{1}, p_{2},-1\right)$ como na Figura 1. Esta interpretação propõe o seguinte: por cada vetor tangente a uma curva inicial e seu vetor normal respectivo, podemos gerar soluções características da função $u$. A existência da solução está garantida pelo teorema de Cauchy.

\section{Teorema De Cauchy}

Seja a equação $F\left(x_{1}, x_{2}, u, u_{x_{1}}, u_{x_{2}}\right)=0$ onde $F \epsilon C^{2}(\Omega), \Omega \subset \mathbb{R}^{2} \times \mathbb{R} \times \mathbb{R}^{2}$ e $\left|F_{p_{1}}\right|+\left|F_{p_{2}}\right|>0$ $\left(x_{1}^{0}(t), x_{2}^{0}(t)\right)$ e $u^{0}(t)$ definidas em $I \subset \mathbb{R}$ tem segunda derivada continua em $I$, satisfazem as seguintes condições

$$
\left\{\begin{array}{c}
\frac{d u^{0}}{d t}(t)=\frac{d x_{1}^{0}}{d t} p_{1}^{0}(t)+\frac{d x_{2}^{0}}{d t} p_{2}^{0}(t) \\
F\left(x_{1}^{0}(t), x_{2}^{0}(t), u^{0}(t), p_{1}^{0}(t), p_{2}^{0}(t)\right)=0
\end{array}\right.
$$

e

$$
\operatorname{det}\left[\begin{array}{cc}
\frac{d x_{1}^{0}}{d t} & \frac{d x_{2}^{0}}{d t} \\
F_{p_{1}}\left(x_{1}^{0}, x_{2}^{0}, u^{0}, p_{1}^{0}(t), p_{2}^{0}(t)\right) & F_{p_{2}}\left(x_{1}^{0}, x_{2}^{0}, u^{0}, p_{1}^{0}(t), p_{2}^{0}(t)\right)
\end{array}\right] \neq 0
$$

então existe uma única função $\phi$ definida por $\phi: G \rightarrow \mathbb{R}, G \subset \mathbb{R}^{2}$ que verifica

$$
\left\{\begin{array}{c}
F\left(x_{1}, x_{2}, \phi\left(x_{1}, x_{2}\right), \phi_{x_{1}}\left(x_{1}, x_{2}\right), \phi_{x_{2}}\left(x_{1}, x_{2}\right)\right)=0 \forall\left(x_{1}, x_{2}\right) \epsilon G \\
\phi\left(x_{1}^{0}(t), x_{2}^{0}(t)\right)=u^{0}(t) \\
\phi_{x_{1}}\left(x_{1}^{0}(t), x_{2}^{0}(t)\right)=p_{1}^{0}(t) \\
\phi_{x_{2}}\left(x_{1}^{0}(t), x_{2}^{0}(t)\right)=p_{2}^{0}(t)
\end{array}\right.
$$

assim $\phi$ é a solução única do problema 


\section{Métodos e Formulação Utilizada}

Pelo teorema de Cauchy e a definição de curvas características é possível obter o seguinte sistema de equações diferenciais ordinária denominado sistema característico

$$
\begin{cases}\frac{d x_{1}}{d s}=F_{p_{1}}, & x_{1}(0)=x_{1}{ }^{0} \\ \frac{d x_{2}}{d s}=F_{p_{2}}, & x_{2}(0)=x_{2}{ }^{0} \\ \frac{d u}{d s}=p_{1} F_{p_{1}}+p_{2} F_{p_{2}}, & u(0)=u^{0} \\ \frac{d p_{1}}{d s}=-F_{x_{1}}-p_{1} F_{u}, & p_{1}(0)=p_{1}{ }^{0} \\ \frac{d p_{2}}{d s}=-F_{x_{2}}-p_{2} F_{u}, & p_{2}(0)=p_{2} u^{0} \\ F\left(x_{1}, x_{2}, u, p_{1}, p_{2}\right)=0 & \end{cases}
$$

Este sistema pode ser resolvido usando o método previsor corretor (Figura 2(a)).Como método previsor usamos a fórmula de Euler, $Y_{i+1}=Y_{i}+h \cdot f(x, t)$, para um sistema de equações diferenciais ordinárias da forma

$$
\left\{\begin{array}{l}
x^{\prime}=f(x, t), \quad t \in[0, b] \\
x^{\prime}(0)=x^{0}
\end{array}\right.
$$

E o método corretor estaria dado pela formula de Newton

$$
P^{k+1}=P^{k}-\frac{F\left(P^{k}\right) \nabla F\left(P^{k}\right)}{\left\|\nabla F\left(P^{k}\right)\right\|}
$$

onde $F: \Omega \subset \mathbb{R}^{2} \times \mathbb{R} \times \mathbb{R}^{2} \rightarrow \mathbb{R}$

Ideias semelhantes foram utilizadas no trabalho [2] para resolver equações integrais baseadas em uma série-homotópica, por sua vez em [3], propõem uma técnica para achar uma solução para EDOs implícitas identificando a curva solução como uma curva implícita sobre uma variedade.

Neste trabalho utilizamos as técnicas de métodos de continuação, propomos achar uma "curva característica" sobre uma variedade e logo utilizar esta curva para achar outras, e assim achar a solução aproximada da equação (1). Uma representação gráfica da solução poderia ser obtida como na Figura 2(b), mediante a triangulação dos pontos obtidos usando para isso alguma técnica conhecida de geração de malhas, como por exemplo Marching Tetrahedra.

\section{Algoritmo Método Previsor Corretor}

Passo 1: Ingressar

- $F\left(x_{1}, x_{2}, u, p_{1}, p_{2}\right) \quad$ : Função implícita

- $C_{0} \quad$ : Curva dado, $\operatorname{com} F\left(C_{0}\right) \approx 0$

- $N_{0} \quad$ : Vetores Normais nos pontos de $C_{0}$

- $t_{0} \quad$ : Ponto inicial do intervalo do domínio da solução numérica

- $b \quad$ : Ponto final do intervalo domínio da solução numérica

- $h \quad$ : Passo inicial

Passo 2: Em quanto $t<b$

$Y_{i+1}=Y_{i}+h \cdot V$ tang $\quad$ Vtang Função que acha a solução do sistema característico (2)

Passo 2.1 Enquanto Erro $>$ tol

$$
\begin{aligned}
& y_{i+1}=y_{i}-\frac{F\left(P^{k}\right) \nabla F\left(P^{k}\right)}{\left\|\nabla F\left(P^{k}\right)\right\|} \\
& \text { Erro }=\left\|y_{i+1}-y_{i}\right\|
\end{aligned}
$$

Fim Passo 2.1

$t=t+h$

Fim Passo 2 


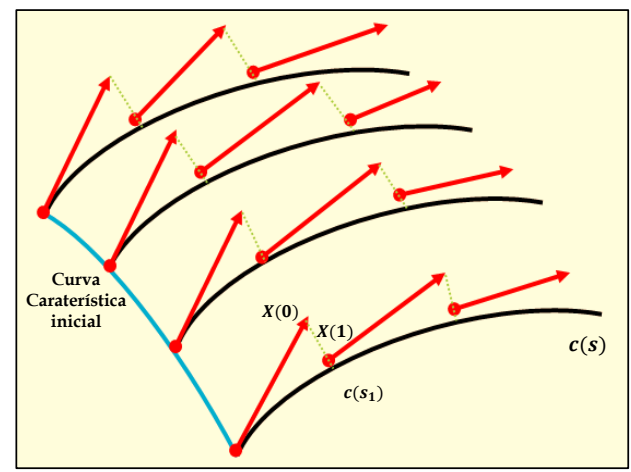

(a) Método Newton e previsor corretor

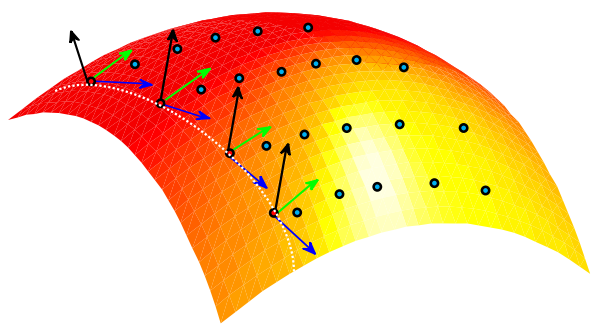

(b) Gráfico da solução

Figura 2: Geração da Superfície usando uma curva característica (solução inicial)

\section{Resultados obtidos}

Como ferramenta na implementação foi utilizada o MATLAB e os resultados são apresentados nas Figuras 3,4,5, que correspondem às seguintes equações.

Primeiro Caso. Um paraboloide hiperbólico $u=x^{2}-y^{2}$ pode expressar-se em termos de uma equação diferencial parcial de primeira ordem tal como

$$
F\left(x_{1}, x_{2}, u, p_{1}, p_{2}\right)=p_{1}+p_{2}-2\left(x_{1}-x_{2}\right)=0
$$

Logo, tomando uma curva dado parametrizada $c(t)=\left(t, 0, t^{2}\right)$ e os vetores normais nesta curva dado $N(t)=(2 t, 0,-1)$, obtemos a solução gráfica da Figura 3 .

Segundo Caso. Um paraboloide Elíptico $u=x^{2}+y^{2}$ pode expressar-se em termos de uma equação diferencial parcial de primeiro ordem tal como

$$
F\left(x_{1}, x_{2}, u, p_{1}, p_{2}\right)=p_{1}+p_{2}-2\left(x_{1}+x_{2}\right)=0
$$

Logo, tomando uma curva dado parametrizada $c(t)=\left(t, 0, t^{2}\right)$ e os vetores normais nesta curva dado $N(t)=(2 t, 0,-1)$, obtemos a solução gráfica da Figura 4.

Terceiro Caso. Uma Esfera $x^{2}+y^{2}+z^{2}=9$ pode expressar-se em termos de uma equação diferencial parcial de primeiro ordem tal como

$$
F\left(x_{1}, x_{2}, u, p_{1}, p_{2}\right)=x_{1}+x_{2}+u p_{1}+u p_{2}=0
$$

Logo, tomando uma curva dado parametrizada $c(t)=(3 \cos (t), 0,3 \sin (t))$ e os vetores normais nesta curva dado $N(t)=(3 \cos (t), 0,3 \sin (t))$, obtemos a solução gráfica da figura 5 .

\section{Conclusões}

O objetivo do método desenvolvido é obter soluções numéricas de EDPs implícitas, para isto, tendo condições iniciais na Equação (1), podemos construir aproximações para as soluções características de $u$ utilizando técnicas semelhantes às propostas em [5]. Neste trabalho associa-se uma equação diferencial ordinária implícita de primeira ordem para uma superfície $S$, desta forma o problema consistiria em resolver um sistema não linear, numa forma semelhante, ao que pretendemos utilizar. Estas técnicas empregam o método de continuação numérica usando o método de Newton modificado. 

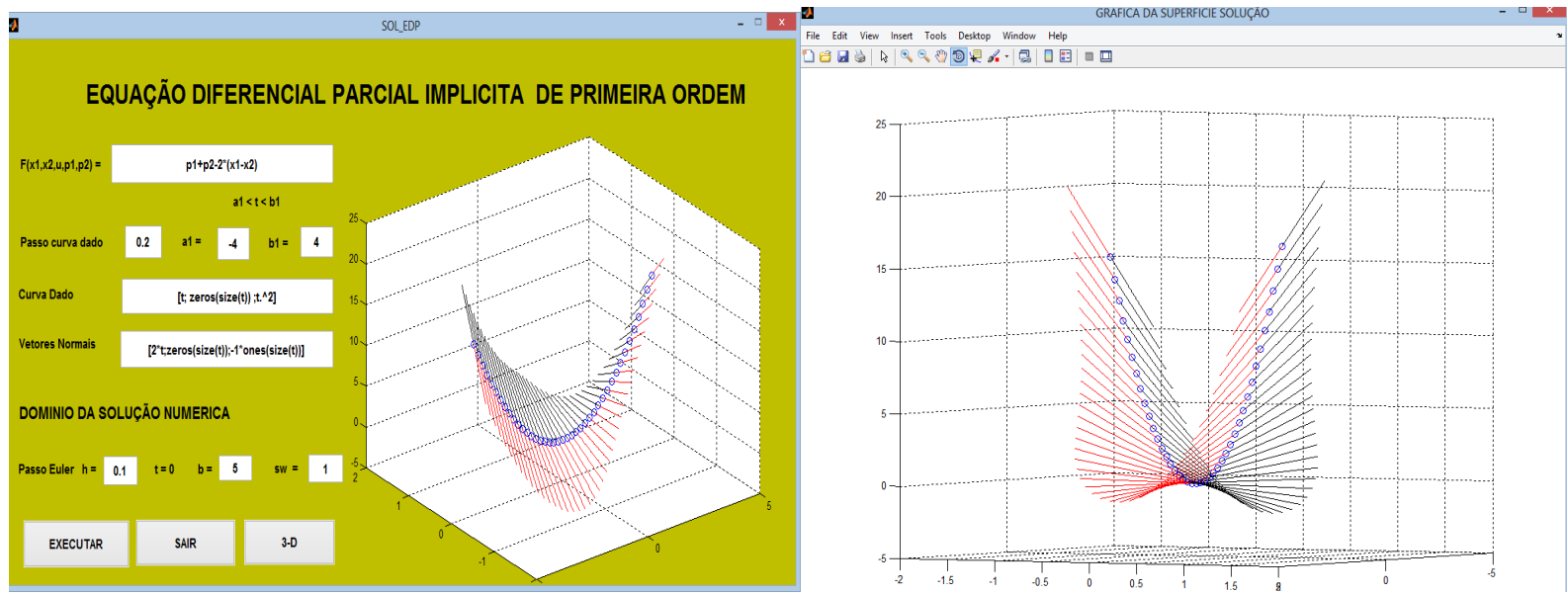

Figura 3: Paraboloide Hiperbólico
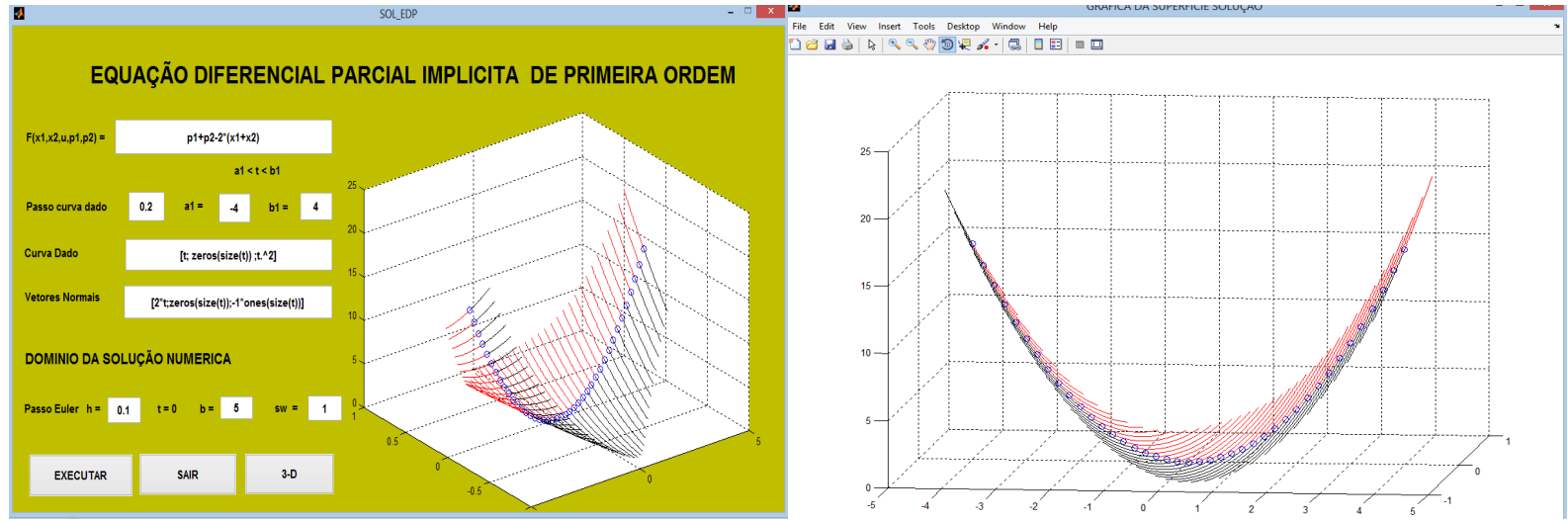

Figura 4: Paraboloide Elíptico

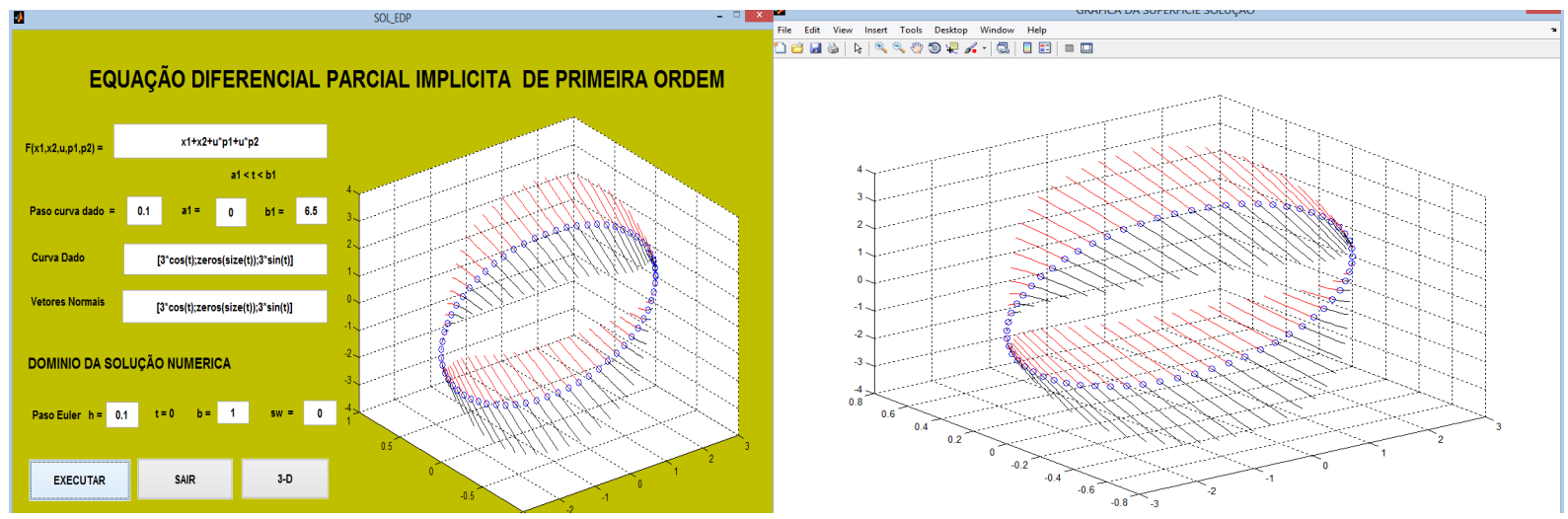

Figura 5: Esfera 
Dada a forma do problema resolvido no caso da equação $F\left(x_{1}, x_{2}, u, p_{1}, p_{2}\right)=0$, notamos que é possível estender ao caso geral para uma equação na forma $F(x, u, \nabla u)=0$ onde $F \in C^{2}(\Omega)$, $\Omega \subset \mathbb{R}^{n} \times \mathbb{R} \times \mathbb{R}^{n}$ a função $u$ ficaria definida como $u: V \rightarrow \mathbb{R}, V \subset \mathbb{R}^{n}$ e $\Omega^{*}$ representará a projeção de $\Omega$ sobre $\mathbb{R}^{n+1}$.

O custo computacional é alto, devido a que em cada etapa se aplicam para cada ponto os métodos previsor corretor. O custo computacional aumenta progressivamente com cada iteração, por que o passo ou a tolerância são reduzidos para alcançar uma melhor precisão, uma parte deve-se à utilização do MATLAB sendo recomendável mudar a outra linguagem como: $\mathrm{C}, \mathrm{C}++$. O método precisa de uma curva inicial e de vetores normais dessa curva, em geral os métodos que resolvem EDPs precisam de uma curva dado inicial, mas não dos vetores normais.

A definição da derivada, é utilizada como uma ferramenta para analisar as variações de magnitude, seu conceito é adaptável em todas as áreas das ciências, especialmente nos fenômenos físicos, onde tem sido gerados muitos modelos baseados em equações diferenciais, podemos mencionar alguns deles: O estudo das equações diferenciais parciais que envolvem fluidos incompressível na atualidade é importante não só no aspecto teórico sino também nas simulações numéricas, dentro todos os modelos desenvolvidos destacam os modelos de Euler e Navier-Stokes. Os modelos de fenômenos de propagação de ondas e vibrações são dados por equações diferenciais parciais de segundo ordem, que a sua vez tem definidas nelas equações de transporte linear, por exemplo, na equação

$$
u_{t}+u_{x}=0
$$

as soluções são ondas viajantes ao longo do tempo.

O trabalho propõe um método numérico para os casos em que a EDP de primeira ordem seja implícita, pois em outros casos existe teoria desenvolvida ao respeito.

\section{Referências}

[1] Eugene. Allgower and Kurt Georg, Introduction to Numerical Continuation Methods, Society for Industrial and Applied Mathematics, 2003.4

[2] F. Awawdeh, A. Adawi and S. Al-SharA', A Numerical Method for Solving Nonlinear Integral Equations. International Mathematical Forum, 4, 2009, no. 17, 805 - 817.

[3] A. Castelo, S. De Freitas and G. Tavares, PL Approximation to Manifolds and Its Application to Implicit ODEs. Lectures in Applied Mathematics, Volume 26, Computational Solution of Nonlinear Systems of Equations. Eugene L. Allgower, Kurt Georg Editors, American Mathematical Society 1990.

[4] R. FAro. Apuntes de Ecuaciones diferenciales, Dpto. de Matemáticas. Univ. de Extremadura Badajoz, 5 de febrero de 2013.

[5] S.R. De Freitas and G. Tavares, Solving implicit ODEs by simplicial methods, in Curves and Surfaces, P.L. Lauret, A. L. Méauté, L.L. Schumaker (eds.), (1991), 193-196.

[6] A.I.Peral. Primer Curso de Ecuaciones en Derivadas Parciales. Editorial Wesley Iberoamericana, Wilmington, Delaware, E.U.A, 1995.

[7] H. Rhee, Rutherford Aris and Neal R. Amundson, Firts-Order Partial Differential Equations, Prentice Hall 1986. 